\title{
VLBI at APEX: First Fringes
}

\author{
A. L. Roy*, J. Wagner, M. Wunderlich, A. Bertarini, T. P. Krichbaum, W. Alef, \\ Max-Planck-Institut für Radioastronomie, Auf dem Hügel 69, 53121 Bonn, Germany \\ E-mail: aroy@mpifr-bonn.mpg.de \\ G. Wieching, C. Duran, R. Olivares, P. Caro, O. Arriagada, \\ F. M. Montenegro-Montes, J. P. Araneda, \\ European Southern Observatory, Alonso de Córdova 3107, Vitacura, Santiago, Chile
}

\section{Lindqvist, R. Haas, J. Johansson, H. Olofsson, M. Pantaleev,}

Onsala Space Observatory, Chalmers University of Technology, Observatorievägen 90, 43992 Onsala, Sweden

\section{R. Freund, D. Marrone, P. Strittmatter, L. Ziurys,} Arizona Radio Observatory, Steward Observatory, University of Arizona, 933 North Cherry Avenue, Tucson, AZ 85721, USA

\section{R. Blundell, R. Primiani, J. Weintroub, K. Young,}

Harvard-Smithsonian Center for Astrophysics, 60 Garden Street, Cambridge, MA 02138, USA

\section{G. Crew, S. Doeleman, V. Fish, R.-S. Lu, J. SooHoo, M. Titus}

MIT Haystack Observatory, Route 40, Westford, MA 01886, USA

\section{G. Tuccari}

Istituto di Radioastronomia, Contrada Renna Bassa, 96017 Noto, Italy

\begin{abstract}
We have equipped the APEX telescope for $1 \mathrm{~mm}$ VLBI and obtained first fringes on 3C 279 at $229 \mathrm{GHz}$ in May 2012 with SMA (Hawaii) and SMTO (Arizona). The fringe spacing achieved was 29 microarcseconds, adequate to directly observe strong-field general-relativistic effects around the black hole in the Galactic center by resolving the expected diameter of the shadow of the event horizon in $\mathrm{Sgr} \mathrm{A}^{*}$ of $\sim 40$ microarseconds. I present on behalf of the collaboration the unusual aspects of this high-altitude VLBI installation, and the prospects for upcoming observations with a global array at the highest resolution.
\end{abstract}

11th European VLBI Network Symposium \& Users Meeting,

October 9-12, 2012

Bordeaux, France

\footnotetext{
* Speaker.
} 


\section{Introduction}

The APEX (Atacama Pathfinder Experiment) telescope, inaugurated in September 2005, is located in the Atacama desert in Chile close to the ALMA site at an altitude of $5104 \mathrm{~m}$. It was built and has been operated by the Max Planck Institute for Radio Astronomy in Bonn (50\%), the Onsala Space Observatory (23\%), and the European Southern Observatory (27\%). The antenna is a modified ALMA prototype manufactured by VERTEX Antennentechnik.

APEX is presently the only telescope in the southern hemisphere available for VLBI at wavelengths of $1 \mathrm{~mm}$ and shorter. Work has started to phase the ALMA antennas to work as a very sensitive VLBI element [1], but ALMA will not become available for VLBI before 2014. Plans exist also to equip the South Pole Telescope for VLBI, but it does not yet have a heterodyne receiver.

The APEX contribution to VLBI arrays observing at $1 \mathrm{~mm}$ and below is quite substantial for all sources with declinations $\lesssim 40^{\circ}$ with respect to north-south resolution (see Figure 1). The great interest in having a southern $1 \mathrm{~mm}$ VLBI-capable antenna is due to the fact that the resolution of such an array is becoming comparable to the event horizons of the nearest active galactic nuclei at $1 \mathrm{~mm}$ wavelength and below. The sources of prime interest here are Sgr A* and M87 with similar $10 \mu$ as and $7.9 \mu$ as Schwarzschild radii. These sources profit most from a southern VLBI antenna due to their low declination. For instance the fringe spacing achieved in our first successful observation was $29 \mu$ as, adequate to resolve the expected diameter of the shadow of the event horizon in Sgr A* of $40 \mu$ as $(0.3 \mathrm{AU})$ and probe strong general relativity at the Galactic centre.

\section{The APEX VLBI project}

Because of its unique location it was realized soon that APEX can make unique contributions to $1 \mathrm{~mm}$ VLBI. The actual planning to equip APEX for VLBI started in March 2009. Initial planning estimated 180 man-weeks (4.1 FTE). The actual effort is currently closer to 500 man-weeks (11 FTE), with a total hardware budget in excess of $500000 €$.
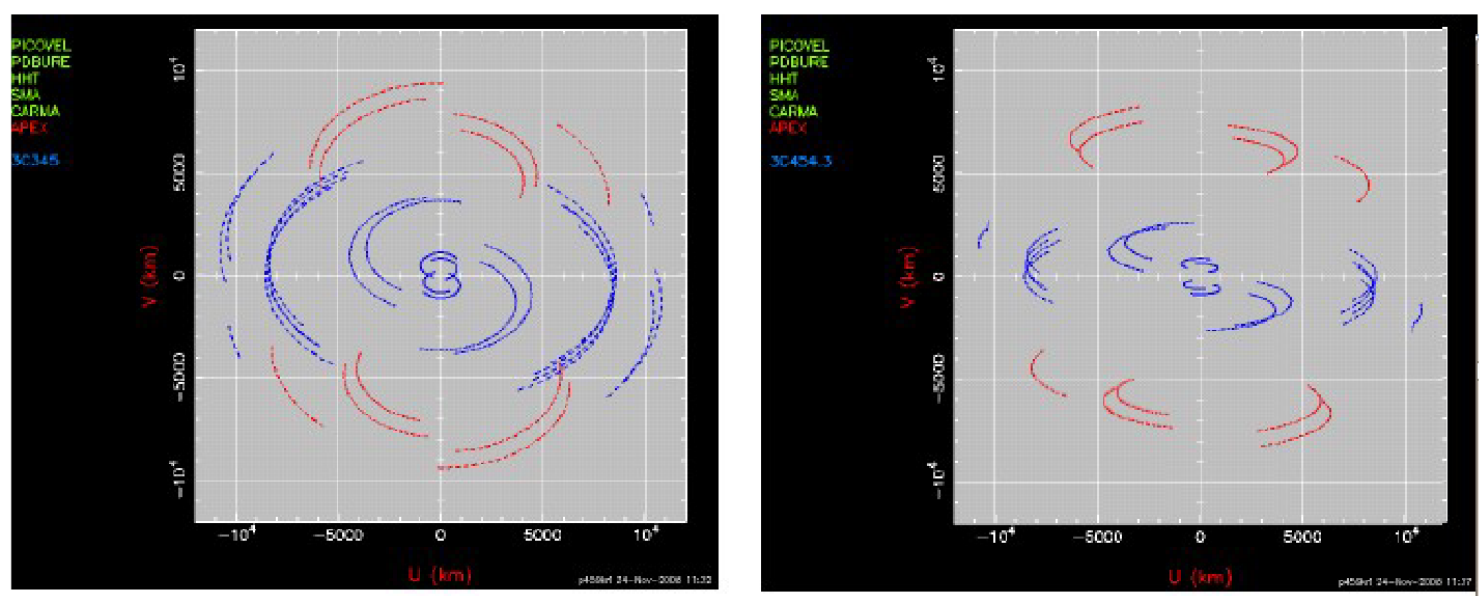

Figure 1: UV-coverage of the mm-VLBI array made up of Pico Veleta, Plateau de Bure, SMTO, CARMA, SMA and APEX. Left: declination $40^{\circ}$, right: declination $16^{\circ}$. The APEX contribution shown in red improves image sharpness and quality significantly. 


\subsection{Installation}

In March of 2010 a hydrogen maser (iMaser 3000 by T4Science) was installed. Due to lack of space and the need for a vibration-free concrete foundation it could not be located inside the containers which serve for housing computers, workshops, storage, and the control room. Finally a semi-open space on the side of a container under a roof was selected. As this location is subject to the large temperature changes of the Atacama desert at $5100 \mathrm{~m}$, a climate chamber designed and manufactured by the company KlimaSystems (Nümbrecht, Germany) for $34200 €$ (15\% of the cost of the maser) was installed. It provides a temperature stability of $\pm 0.1^{\circ} \mathrm{C}$ over $-20^{\circ} \mathrm{C}$ to $+30^{\circ} \mathrm{C}$, humidification (the air in the desert is extremely dry), and magnetic shielding. Its footprint is $1.5 \mathrm{~m}$ by $1.5 \mathrm{~m}$ and it has a vibrationally decoupled air conditioning unit $(1.0 \mathrm{~m} \times 0.75 \mathrm{~m})$.

In March 2011 a Mark 5C recorder with a SSD system disk was brought to the site. As the disks in Mark 5 modules fail quickly at an altitude of $5100 \mathrm{~m}$, a specially designed pressure chamber was procured from Reichert GmbH, Bonn (replication cost $\sim 25000 €$ ). The chamber has an altitude downrated cooling capacity of $1.5 \mathrm{~kW}$ and an internal pressure of $200 \mathrm{hPa}$ above the ambient pressure which at APEX corresponds to a safe disk operating altitude of $3000 \mathrm{~m}$. The disks are keyed by a microcontroller after pressure exceeds a threshold of $200 \mathrm{hPa}$ to prevent them from spinning while at too low pressures. The housing fits two recorders and four disk modules.

The DBBC backend [2] had to be installed at the telescope close to the receiver, while the Mark 5C recorder had to be placed in one of the control containers due to lack of space. As this exceeded the $\sim 2 \mathrm{~m}$ reach of the copper $10 \mathrm{GbE} \mathrm{CX} 4$ interface of the Mark 5C, additional new hardware was needed. A new interface (FiLa10G) was added to the DBBC which translates 32 or 64 LVDS pairs (DBBC VSI outputs) into a UDP/IP data stream, sent at up to $4 \mathrm{Gbit} / \mathrm{s}$ using a standard optical short-range $10 \mathrm{GbE} \mathrm{XFP}$ transceiver. The data are carried over a $100 \mathrm{~m}$ optical fibre (OM3 50/125 $\mu \mathrm{m}$; laid in 2010) down to the control container where a newly developed fibreto-copper media converter (Glapper; SR XFP to CX4) brings the data to the Mark 5C recorder.

Long coaxial cable runs were needed to connect e.g. the H-maser, the frontend and the DBBC with 1 PPS and $10 \mathrm{MHz}$ signals. For these we selected Times Microwave LMR-200 for its low temperature coefficient $\left(\sim 25 \mathrm{ppm} /{ }^{\circ} \mathrm{C}\right.$; Norrod 2003, NRAO Internal Report).

Another item installed in 2011 was a downconverter designed to mix the APEX IF to the frequency range the DBBC can handle. Amongst the many more components which are necessary for reliable VLBI operation (see Fig. 2) the test tone generator should be mentioned which was used to monitor the phase stability of the complete signal path from the receiver horn down to the recorded data.

\subsection{Station position}

For VLBI at $345 \mathrm{GHz}$ the station position had to be known with sufficient precision $(<3 \mathrm{~m})$ to limit the residual fringe rates to less than $100 \mathrm{mHz}$. A GPS choke-ring antenna borrowed from TIGO (thanks to H. Hase) was mounted on APEX. It was connected to a dual-frequency GPS receiver from Onsala operating in an APEX instrument container rack. The antenna moved with the telescope which made the antenna rotate on a circle with the azimuth axis as its centre. GPS kinematic position solutions were determined, to which a circle was fit. Uncertainties on the circle centre are: $[0.3,0.2,0.1] \mathrm{mm}$ in $[\mathrm{X}, \mathrm{Y}, \mathrm{Z}]$. We added $1 \mathrm{~cm}$ uncertainty for measuring the height of 


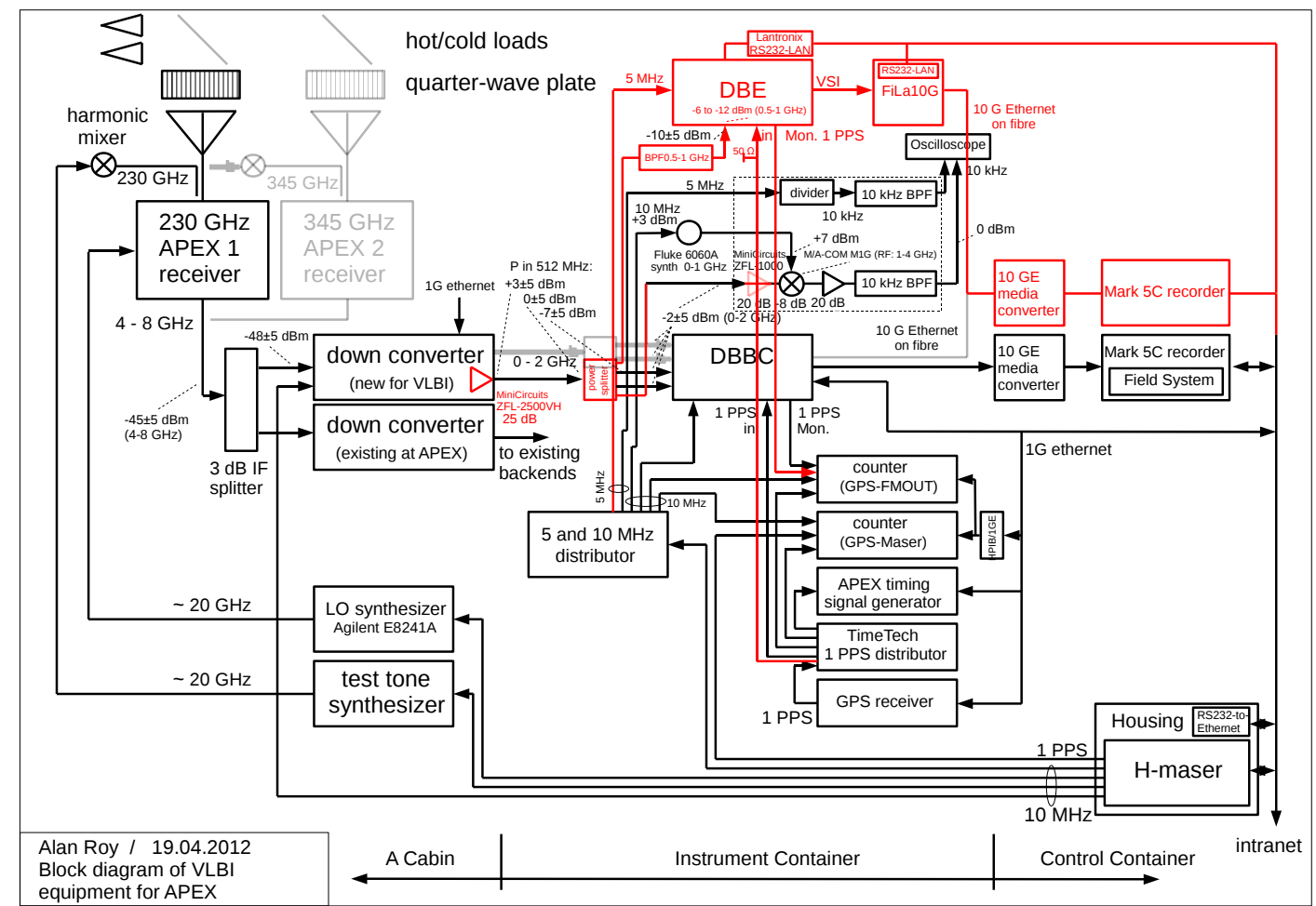

Figure 2: Block diagram of APEX VLBI equipment. In red: temporary equipment brought to APEX in 2012 to verify the backend synchronization.

the telescope elevation axis above the GPS antenna reference plane (see Fig. 3). The final [X, Y, Z] position was [2225039.5297, -5441197.6292, -2479303.3597] at epoch 2011-Mar-26 in the IGS05 reference frame (same as ITRF2005).

\subsection{Receiver phase fluctuations}

When the phase stability of the $230 \mathrm{GHz}$ receiver was measured with a test tone injector before the receiving horn strong phase fluctuations were found, which would degrade the phase coherence for VLBI by $30 \%$. The injector was modified to reduce the blockage of the beam into the receiver to a minimum. The test tone was then recorded with the data continuously during observing. Its phase could be detected in the data in $20 \mathrm{~ms}$ averages with good SNR ( $\mathrm{rms}$ phase noise $2^{\circ}$ ).

Later the fringe-fitting software was modified to use the detected fluctuations of the test tone phase to remove most of the phase instability of the receiver (see Fig. 4).

\section{Failed observations in 2011}

We observed a first fringe test on March 28 to April 2, 2011 with SMTO, CARMA, SMA, and Pico Veleta. Fringes were found on some baselines but unfortunately not to APEX. After careful analysis of logs and on-site measurements we suspect a large clock offset is present. A critical point is the synchronization of all digital electronic components to the station 1 PPS and due to one 


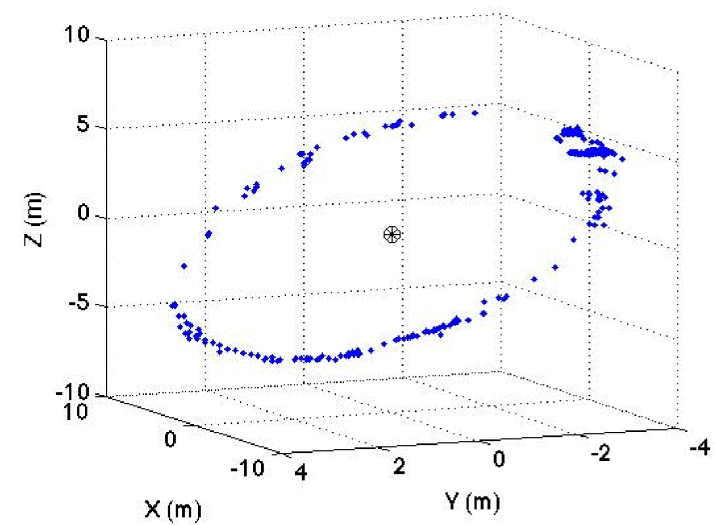

Figure 3: The GPS measurements describe a circle with an uncertainty of the centre of $[0.3,0.2,0.1]$ $\mathrm{mm}$ in $[\mathrm{X}, \mathrm{Y}, \mathrm{Z}]$.

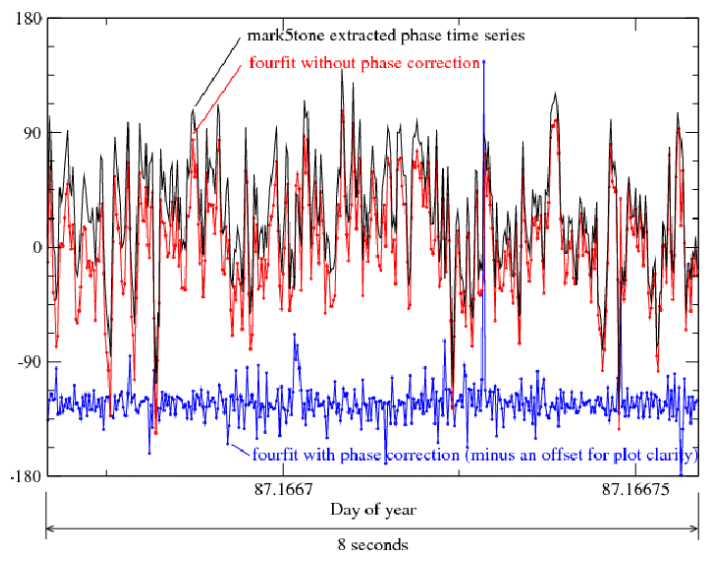

Figure 4: Black: extracted phase of test tone. Red: fringe-fit phase without correction. Blue: corrected phase (offset for plotting).

small cabling error to the GPS-FMOUT counter it was probably not noticed that a backend went out of sync.

The fringe search still continues. Due to the huge search space in clock (and to some degree in residual fringe rate) this is a cumbersome task which takes a lot of manpower and computer resources. Correlations are done with 250000 delays to cover a total of $2 \mathrm{~s}$ of possible clock offset.

\section{First fringes with APEX in May 2012}

To be able to completely verify that the backend works, a second backend (DBE) was borrowed from Haystack. Together with a second Mark $5 \mathrm{C}$ recorder both backends could be checked against each other with a zero-baseline test. Complete debugging of the station led to the discovery of one wrong connection which in 2011 lead to the false diagnosis of a synchronized backend.

In the second fringe test APEX observed with SMTO and SMA and fringes on 3C 279 were found soon after (see Fig. 5). For some time intervals the coherence time was surprisingly large $\sim 30$ s (see Fig. 6), though was typically $\sim 5$ s. First scientific results will be published in [3] and [4].

\section{References}

[1] W. Alef, et al., An ALMA beamformer for ultra high resolution VLBI and phased array science, in Proceedings of 11th EVN Symposium, POS (11th EVN Symposium) 053.

[2] G. Tuccari, Development of a Digital Base Band Converter (DBBC): Basic Elements and Preliminary Results, in: New Technologies in VLBI, Astronomical Society of the Pacific Conference Series, ISSN 1050-3390, Vol. 306, 177-252, 2004.

[3] T. P. Krichbaum, et al., Zooming towards the Event Horizon - mm-VLBI today and tomorrow, in Proceedings of 11th EVN Symposium, PoS (11th EVN Symposium) 055.

[4] J. Wagner, et al. 2014, in preparation. 


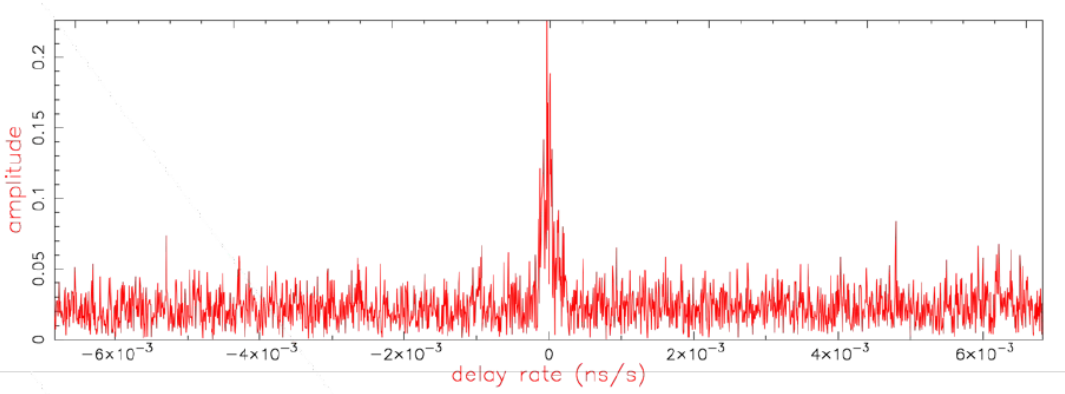

APEX - SMA

SNR $=12.3$

Integration time $=7 \mathrm{~min}$

Residual rate $=7.7 \mathrm{mHz}$

Baseline $=9447 \mathrm{~km}$

Fringe spacing $=\mathbf{2 9}$ uas

(World record resolution)

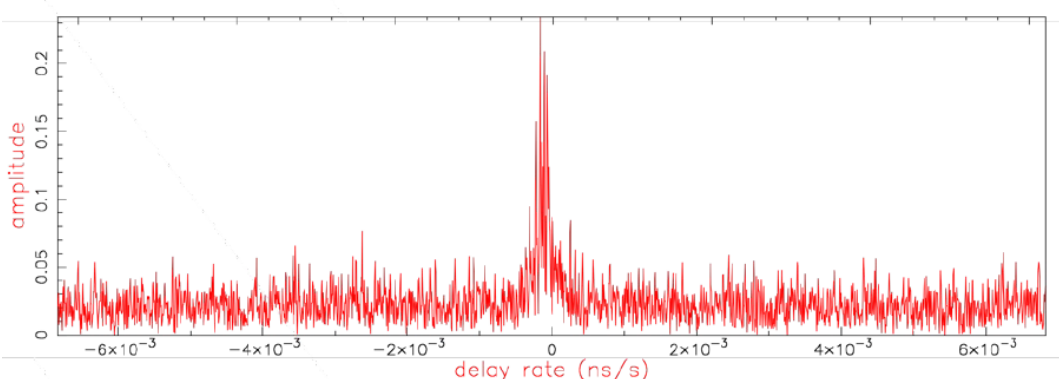

\section{APEX - SMTO}

SNR $=12.7$

Integration time $=7 \mathrm{~min}$

Residual rate $=-37.9 \mathrm{mHz}$

Baseline $=7174 \mathrm{~km}$

Fringe spacing $=\mathbf{3 8}$ uas

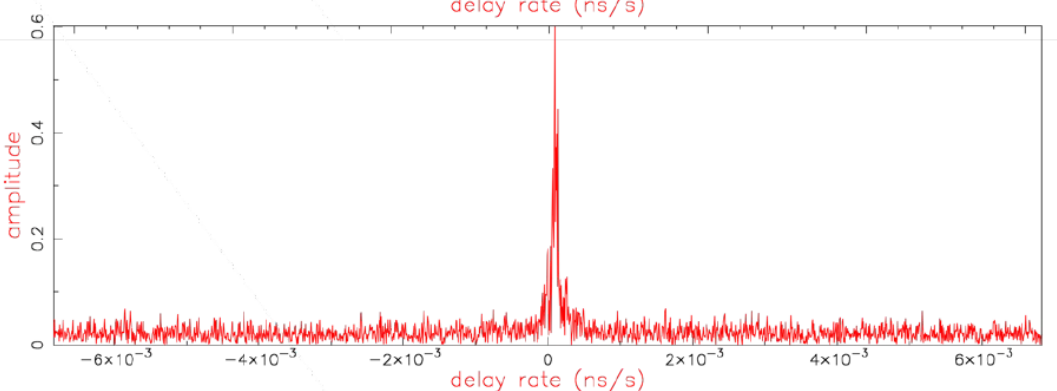

SMA - SMTO

SNR $=32.6$

Integration time $=7 \mathrm{~min}$

Residual rate $=21.4 \mathrm{mHz}$

Baseline $=4627 \mathrm{~km}$

Fringe spacing $=\mathbf{5 8}$ uas

Figure 5: Fringe amplitude as a function of residual fringe-rate for the three baselines in the first successful fringe test with the APEX telescope. A world record in resolution of $29 \mu$ as (about $7 \mathrm{G} \lambda$ ) was achieved on the APEX-SMA baseline.

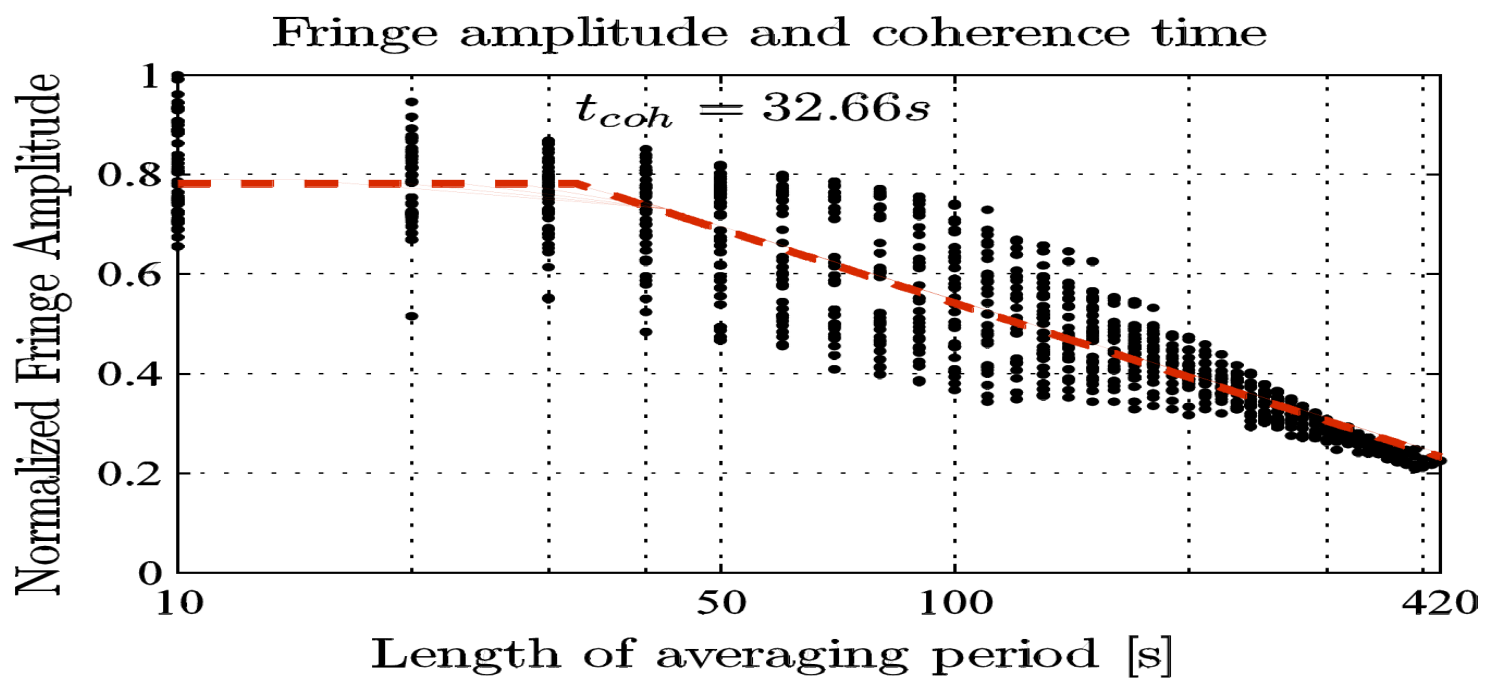

Figure 6: Fringe amplitude as a function of integration time. 